\title{
Post-exposure passive immunisation for preventing measles (Protocol)
}

Young MK, Nimmo GR, Cripps AW, Jones MA
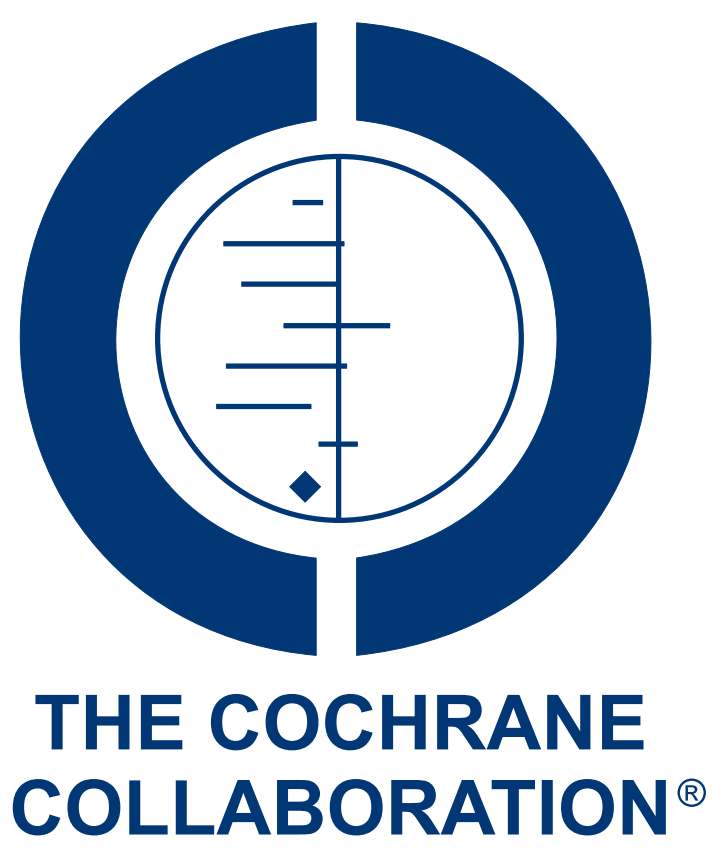

This is a reprint of a Cochrane protocol, prepared and maintained by The Cochrane Collaboration and published in The Cochrane Library 2012, Issue 9

http://www.thecochranelibrary.com

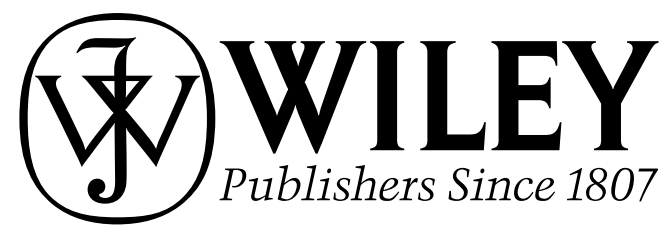

Post-exposure passive immunisation for preventing measles (Protocol)

Copyright $\odot 2012$ The Cochrane Collaboration. Published by John Wiley \& Sons, Ltd. 
TABLE OF CONTENTS

HEADER . . . . . . . . . . . . . . . . . . . . . . . . . . . . . . . . . . . . . . . . 1

ABSTRACT . . . . . . . . . . . . . . . . . . . . . . . . . . . . . . . . . . . . . . 1

BACKGROUND . . . . . . . . . . . . . . . . . . . . . . . . . . . . . . . . . . . . . 2

OBJECTIVES . . . . . . . . . . . . . . . . . . . . . . . . . . . . . . . . . . . . . . .

METHODS . . . . . . . . . . . . . . . . . . . . . . . . . . . . . . . . . . . . . .

ACKNOWLEDGEMENTS . . . . . . . . . . . . . . . . . . . . . . . . . . . . . . . . . . . . . . . .

REFERENCES . . . . . . . . . . . . . . . . . . . . . . . . . . . . . . . . . . . . . . 6

APPENDICES . . . . . . . . . . . . . . . . . . . . . . . . . . . . . . . . . . . . . . 8

HISTORY . . . . . . . . . . . . . . . . . . . . . . . . . . . . . . . . . . . . . . . . 10

CONTRIBUTIONS OF AUTHORS . . . . . . . . . . . . . . . . . . . . . . . . . . . . . . . . . . . . . . . . .

DECLARATIONS OF INTEREST . . . . . . . . . . . . . . . . . . . . . . . . . . . . . . . . . . . . . .

SOURCES OF SUPPORT . . . . . . . . . . . . . . . . . . . . . . . . . . . . . . . . . . . . 


\title{
[Intervention Protocol]
}

\section{Post-exposure passive immunisation for preventing measles}

\author{
Megan K Young ${ }^{1}$, Graeme R Nimmo², Allan W Cripps ${ }^{3}$, Mark A Jones ${ }^{4}$ \\ ${ }^{1}$ School of Medicine, Griffith Health Institute, Griffith University, Meadowbrook, Australia. ${ }^{2}$ Department of Microbiology, Pathology \\ Queensland, Brisbane, Australia. ${ }^{3}$ Griffith Health, Griffith University, Gold Coast, Australia. ${ }^{4}$ Centre for Healthcare Related Infection \\ Surveillance and Prevention, Queensland Health, Brisbane, Australia \\ Contact address: Megan K Young, School of Medicine, Griffith Health Institute, Griffith University, University Drive, Meadowbrook, \\ Queensland, 4121, Australia. megan.young@griffith.edu.au.
}

Editorial group: Cochrane Acute Respiratory Infections Group.

Publication status and date: New, published in Issue 9, 2012.

Citation: Young MK, Nimmo GR, Cripps AW, Jones MA. Post-exposure passive immunisation for preventing measles. Cochrane Database of Systematic Reviews 2012, Issue 9. Art. No.: CD010056. DOI: 10.1002/14651858.CD010056.

Copyright (C) 2012 The Cochrane Collaboration. Published by John Wiley \& Sons, Ltd.

\section{A B S T R A C T}

This is the protocol for a review and there is no abstract. The objectives are as follows:

To assess the effectiveness and safety of intramuscular injection or intravenous infusion of immunoglobulins for preventing measles when administered to exposed susceptible people before the onset of symptoms. 


\section{B A C K G R O U N D}

Before vaccination against measles was available, annual case numbers were estimated at 130 million (WHO 1999), and the disease caused between five and eight million deaths globally each year (Moss 2009). With the introduction of the vaccine, the worldwide number of cases began to decline (WHO 1999) and this trend continued with increasing vaccination coverage (WHO 2009a). However, the number of measles cases worldwide exceeded 300,000 in 2010 (WHO 2011) and measles is still an important cause of global mortality as identified by the joint World Health Organization (WHO) and United Nations International Children's Emergency Fund (UNICEF) Global Immunization Vision and Strategy 2005-2015 (WHO 2005). One of the strategy's goals is measles mortality reduction. In 2008, measles caused around 164,000 deaths (WHO 2009b).

Further to mortality reduction, most WHO regions have set measles elimination goals and reported on progress towards these (Castillo-Solorzano 2011; Martin 2011; Sniadack 2011; WHO 2008). Many countries have noted continued reductions in incidence (WHO 2012a) and even elimination of endemic transmission (Parker Fiebelkorn 2010). However, the WHO cautions that failure to maintain high vaccination coverage in all areas of a country results in resurgence of the disease (WHO 2009a). Certainly, there are many recent published reports of measles outbreaks among countries with high vaccination coverage (CDC 2011a; Delaporte 2011; DVD CDC 2011; Hoskins 2011; Parker Fiebelkorn 2010; Smithson 2010; Takimoto 2011; Vainio 2011) and the WHO confirms that the incidence of measles worldwide increased in 2010 because of large outbreaks in some regions (WHO 2012a).

In countries with low incidences of measles, elimination strategies typically include an urgent response to a single reported case, including confirmation of the diagnosis, contact tracing and postexposure prophylaxis (CDC 1998; CDNA 2009; NZ MoH 2011; UK DoH 2010). Post-exposure prophylaxis may be a vaccination, which seems to be effective at preventing disease onset if administered within 72 hours of exposure (Barrabeig 2011), or may involve passive immunisation with immunoglobulin, particularly if outside this 72-hour window (Heymann 2008).

\section{Description of the condition}

Measles is a highly communicable viral illness (Heymann 2008). The measles virus is an enveloped, single-stranded RNA Morbillivirus of the family Paramyxoviridae (Heymann 2008; WHO 2009a). The virus is shed from the respiratory tract of infected persons and transmitted by aerosolised droplets or by direct contact with respiratory secretions (WHO 2009a). Someone with measles is contagious from one day before the symptoms start until four days after the rash appears. A susceptible person exposed to measles will usually develop symptoms after around 10 days but this may range from 7 to 18 days after exposure (Heymann 2008).

Symptoms of measles include fever, conjunctivitis, runny nose, cough and a red blotchy rash (WHO 1999). The illness is often more severe in infants and adults than in children (Heymann 2008). Complications occur more frequently in cases in low-income rather than high-income countries (75\% or more versus $10 \%$ to $15 \%$ of cases, respectively) (WHO 1999). Middle ear infection and pneumonia are fairly common complications, occurring in $5 \%$ to $15 \%$, and $5 \%$ to $10 \%$ of children with measles, respectively (WHO 2009a). Encephalitis is a serious, but rarer, complication of measles, occurring in about 1 out of every 1000 cases (WHO 2009a). A slowly progressing neurological disease, subacute sclerosing panencephalitis (SSPE), very rarely (1 out of 100,000 cases) occurs several years after the original measles infection, most often in children infected with measles under the age of two years (Heymann 2008).

\section{Description of the intervention}

The practice of passive immunisation against measles has been used since the 1930s (Zingher 2005). Polyclonal immunoglobulins are administered parenterally to susceptible individuals, who have been in contact with an infectious case of measles, in an attempt to prevent the onset of disease or modify disease expression (Keller 2000).

Immunoglobulin preparations were originally made from animal sera but today are made from pooled donated human plasma (Gonik 2011). Both intramuscular and intravenous preparations are in use. Product names vary from country to country; so too the concentration of disease-specific immunoglobulins in the products will generally reflect circulating antibody levels in the donating populations (Sawyer 2000). Although, in some countries, minimum neutralising antibody concentrations to measles may be regulated (Sawyer 2000).

Current recommendations for dose calculations vary by country, although they are all calculated according to body weight (CDC 2011b; CDNA 2009; ID HPA 2009; NZ MoH 2011). Regardless of the dose recommended, passive immunisation is not currently recommended if more than six days have elapsed since exposure to measles (CDC 2011b; CDNA 2009; ID HPA 2009; NZ MoH 2011).

\section{How the intervention might work}

Whether injected or infused, the administered immunoglobulins distribute throughout the recipient's body (Birdsall 2009). The mechanism by which the recipient is protected from disease involves interaction between the immunoglobulins, the invading measles virus particles and the cells and molecules of the recipient's immune system (Reading 2007). The exact mechanisms by which 
viral infectivity is mitigated by antibodies within the body are not comprehensively understood, but vary according to the structure and functionality of the particular antibodies as they encounter the particular virus particles (Reading 2007). In general, measlesspecific antibodies bind to invading measles virus particles and this may prevent their entry into cells directly, or trigger other immune mechanisms that result in neutralisation or destruction of the virus (Birdsall 2009; Keller 2000; Reading 2007).

\section{Why it is important to do this review}

The effectiveness of post-exposure prophylaxis against measles with immunoglobulins is generally accepted (ATAGI 2008; CDC 1998; NZ MoH 2011; Ramsay 2009). However, effectiveness rates vary considerably among identified reports (King 1991; Ordman 1944; Sheppeard 2009; Stokes 1944).

Further, national recommendations for the use of post-exposure immunoglobulins for measles differ across a number of Western countries (Best 2011; CDC 1998; CDNA 2009; ID HPA 2009; NZ MoH 2011; Ramsay 2009). Disease incidences (WHO 2012b), immunisation schedules (ATAGI 2008; Gustavo 2008; HPA 2011; NZ MoH 2011), measles-containing vaccine coverage (WHO 2012b) and relevant literature are similar. Differences in immunoglobulin dosage recommendations may reflect differences in the minimum levels of measles-specific antibodies in intramuscular preparations (Best 2011; Ramsay 2009; Sawyer 2000).

We could not identify any systematic review evidence of the effectiveness of post-exposure passive immunisation against measles, nor any systematic review evidence of the minimum effective dosage of immunoglobulin for post-exposure prophylaxis against measles. Recent guidance from the United Kingdom on the required dosage of intramuscular immunoglobulin is based on a single study (Endo 2001; Ramsay 2009).

A systematic review of the evidence of effectiveness of passive immunisation for the post-exposure prophylaxis of measles would help to clarify the effectiveness rate and the minimum effective dose and identify differences in benefit or harm across population groups. These outcomes would be valuable to guide public health practice in countries with low incidences of measles.

\section{O B JECT IVES}

To assess the effectiveness and safety of intramuscular injection or intravenous infusion of immunoglobulins for preventing measles when administered to exposed susceptible people before the onset of symptoms.

\section{METHODS}

\section{Criteria for considering studies for this review}

\section{Types of studies}

We will include randomised controlled trials (RCTs), quasi-RCTs and prospective non-RCTs (cohort studies), irrespective of blinding, publication status, language or unit of randomisation. We will include prospective non-RCTs given that more recent studies, using current immunoglobulin preparations, are likely to be nonrandomised for ethical reasons. The intervention has been part of public health practice since the 1930s and as such, any RCTs are likely to have been conducted at a time when the antibody levels of blood donors were due to infection with measles rather than vaccination. To inform practice appropriately, any evidence of the effectiveness of current immunoglobulin preparations should be included.

\section{Types of participants}

People of any age, sex or ethnic origin who were susceptible (no history of measles and not vaccinated against measles and/or measles immunoglobulin $\mathrm{G}$ ( $\mathrm{IgG}$ ) negative) and exposed to measles virus or exposed to someone diagnosed with measles, and who were asymptomatic at the time of intervention or control administration. The primary study's definition of 'exposed' will be accepted and any differences explored via subgroup analysis.

\section{Types of interventions}

1. Intervention: intramuscular injection of polyclonal immunoglobulins; intravenous infusion of polyclonal immunoglobulins.

2. Control: no intervention or placebo or live attenuated measles virus vaccine.

We will also include studies assessing different brands or preparations of polyclonal immunoglobulins or different dosages of immunoglobulins. We will only include studies where the intervention (and control) are administered to participants after exposure to measles and before the participants develop measles symptoms.

\section{Types of outcome measures}

\section{Primary outcomes}

1. Cases of measles. The diagnosis may be made by detection or isolation of measles virus in urine or respiratory secretions; by detection of measles virus antigen in urine or respiratory secretions; by serological detection of immunoglobulin M (IgM) to measles in the absence of vaccination eight days to eight weeks prior to testing; by IgG seroconversion or by a fourfold or greater rise in titre to measles virus in the absence of vaccination eight days to eight weeks prior to testing; or by symptoms consistent 
with measles (fever, a red blotchy rash, conjunctivitis, runny nose and cough) or modified measles (prolonged incubation period, milder fever, cough, runny nose, conjunctivitis and sparse discrete rash of short duration).

2. Mortality due to measles.

\section{Secondary outcomes}

1. Prevention of measles outbreak (higher than expected incidence) as identified by active surveillance.

2. Cessation of measles outbreak (return to expected incidence) as identified by active and/or passive surveillance.

3. Complications due to measles such as otitis media, pneumonia or encephalitis.

4. Occurrence and type of adverse events. We will analyse two types of adverse events: serious adverse events and non-serious adverse events. A serious adverse event is "any untoward medical occurrence that at any dose results in death, is life-threatening, requires inpatient hospitalisation or prolongation of existing hospitalisation, results in persistent or significant disability/ incapacity, or is a congenital anomaly/birth defect" (EMEA 1995). We will classify all other events as non-serious. We will specifically extract data on: blood-borne virus infection; anaphylaxis; generalised hypersensitivity; and injection site reactions. We will also include any other adverse event reported as such by study authors.

\section{Search methods for identification of studies}

\section{Electronic searches}

We will search the Cochrane Central Register of Controlled Trials (CENTRAL) (The Cochrane Library, current issue at the time), which contains the Cochrane Acute Respiratory Infections (ARI) Group's Specialised Register, MEDLINE (1946 to present), CINAHL (1981 to present) and EMBASE (1980 to present). We will use the search strategy in Appendix 1 to search MEDLINE and CENTRAL. We will adapt the strategy for the other databases. We will combine the MEDLINE and CENTRAL searches with the filter for study type in Appendix 2 only if the search results retrieved are too large to be manageable. We will adapt the filter as necessary for the other databases.

\section{Searching other resources}

We will search reference lists of identified relevant studies and reviews. To locate further published or unpublished studies, we will attempt to contact companies manufacturing immunoglobulin products for countries with low measles incidences and attempt to contact the corresponding author of any included studies.

\section{Data collection and analysis}

\section{Selection of studies}

Two review authors (MY, GN) will independently inspect the title and abstract (as available) of each reference identified by the search and determine the potential relevance of each article. If identified by either review author as potentially relevant, we will retrieve the full article. Both review authors will inspect each full article independently, using an eligibility checklist based on the inclusion criteria, to determine inclusion in the review. We will resolve any disagreements through discussion, or by consulting a third review author (AC). We will exclude studies not meeting the eligibility criteria and we will state the reasons for exclusion.

We will list duplicate publications with the main publication for included studies. We will attempt to write to corresponding authors if uncertainties about duplicate publications exist.

\section{Data extraction and management}

Two review authors (MY, AC) will independently extract data from the included studies using pre-designed data extraction forms. We will resolve disagreement by discussion, or by a third review author (GN), independently extracting the data, as necessary. We will attempt to contact study authors for clarification or further information as necessary.

We will extract the following data.

1. The study

i) First author, publication year/not published.

ii) Location.

iii) Date study undertaken.

iv) Randomised/quasi-randomised/non-randomised.

2. Participants

i) Number in each group.

ii) Age range in each group.

iii) Proportion of adults, children, infants (aged < one year) in each group.

iv) Gender distribution in each group.

v) Proportion of high risk individuals in each group:

those with immunodeficiency; pregnancy or age under one year.

vi) Range of time since exposure in each group.

vii) Average time since exposure in each group.

viii) Any measure of baseline comparability and result of this, if calculated.

3. Intervention

i) Intervention group: product used, concentration of measles antibody if known, volume given, route of administration.

ii) Control group: placebo/vaccine/product/other, concentration of measles antibody if relevant and known, volume given, route of administration.

4. Outcomes

i) Primary and secondary (as above). 
ii) Length of follow-up.

iii) Loss to follow-up.

\section{Assessment of risk of bias in included studies}

Two review authors (MY, AC) will independently assess the risk of bias for included studies. We will resolve any disagreements by discussion or by including a third review author (GN) as needed. For randomised and quasi-randomised studies, we will assess: randomisation sequence generation; allocation concealment; blinding of participants, personnel and outcome assessors; incomplete outcome data; drop-out/selective reporting; and other potential sources of bias. We will report the risk of bias using The Cochrane Collaboration's tool for assessing 'Risk of bias' (Higgins 2011). For non-randomised studies, allocation concealment is not applicable. We will therefore assess: blinding of participants, personnel and outcome assessors; incomplete outcome data; drop out/ selective reporting; group differences at baseline; management of confounders and other potential sources of bias. We will report the risk of bias using a modified version of the risk of bias tool (Higgins 2011).

\section{Measures of treatment effect}

Outcomes, as identified above, are dichotomous. We will express these outcomes as risk ratios (RRs) and calculate 95\% confidence intervals (CIs) for each.

\section{Unit of analysis issues}

Should cluster-randomised trials be included in the review, we will attempt to extract RRs and 95\% CIs resulting from analyses that have accounted for the clustering directly from the paper(s). If this is possible, we will proceed to meta-analyse the data using the inverse variance method. If this is not possible, we will extract the number of clusters, the average size of each cluster, the outcome data at the level of the individuals and an estimate of the intracluster correlation coefficient and proceed to reduce the trial(s) to their 'effective sample size' for meta-analysis (Higgins 2011).

Should studies with multiple intervention groups, for example different doses of immunoglobulins compared to control, be included in the review, we will calculate a weighted average of the relevant pair-wise comparisons from the study and a variance for the study, taking into account the correlation between the comparisons.

\section{Dealing with missing data}

We will attempt to contact the trial authors for any missing data. Where missing data exceeds $20 \%$, or where data are clearly missing in different proportions in the treatment groups $(\mathrm{P}<0.05)$, we will exclude the study from further assessment of the relevant outcome. For smaller amounts of missing data, we will undertake sensitivity analysis after assuming worst case and best case scenarios.

\section{Assessment of heterogeneity}

We will explore the presence of heterogeneity firstly by comparing studies' population groups and interventions. If heterogeneity is clear, we will not proceed to meta-analysis. If there is no obvious heterogeneity, we will proceed to meta-analysis and consider the forest plot for each primary outcome and the secondary outcome, "serious adverse events". If statistical heterogeneity is clear visually, we will proceed to subgroup and sensitivity analyses and reexamine the heterogeneity of these results separately. If heterogeneity is not obvious in the initial forest plots, we will consider the Cochrane $\mathrm{Chi}^{2}$ test and $\mathrm{I}^{2}$ statistic for each outcome. We will consider an $\mathrm{I}^{2}$ estimate of $60 \%$ or more, alongside a $\mathrm{Chi}^{2} \mathrm{P}$ value of 0.1 or less important heterogeneity and again proceed to subgroup and sensitivity analyses. If heterogeneity is not explained by subgroup or methodological differences, we will report the results of a random-effects model for the relevant outcome(s).

\section{Assessment of reporting biases}

In the event of multiple publications of the same study, we will list the subsequent papers with the main paper and only enter data once. If uncertainty exists in this respect, we will attempt to contact the study authors. We will assess publication bias by examining funnel plots if we have sufficient studies (at least 10).

\section{Data synthesis}

We will calculate the RR and 95\% CI for each outcome measured in each study. We will use the fixed-effect model for each primary outcome and the secondary outcome, "serious adverse events" and examine the forest plots to assess heterogeneity. We will then explore possible reasons for apparent heterogeneity via subgroup and sensitivity analyses. If these do not explain the apparent heterogeneity, we will use a random-effects model to analyse the pooled studies and report this result. If heterogeneity is minimal, or explained by subgroup or sensitivity analyses, we will report these using a fixed-effect model.

Even in the absence of apparent significant heterogeneity for the primary outcome of "cases of measles", we will proceed to subgroup and sensitivity analysis if we have a sufficient number of included studies.

We will report the results of the other secondary outcomes descriptively.

\section{Subgroup analysis and investigation of heterogeneity}

For subgroup analysis we will include:

- study type;

- age of participants (infants/children/adults/combinations);

- proportion of high risk individuals (pregnant plus

immunodeficient plus aged under one year);

- dose of immunoglobulins;

- dose of measles-specific immunoglobulins; 
- route of administration of immunoglobulins;

- timing of administration of intervention in relation to exposure; and

- differences in primary study definitions of 'exposed' if necessary.

\section{Sensitivity analysis}

For sensitivity analysis we will include:

- risk of bias of included studies; and
- studies with imputed missing data.

\section{ACKNOW LEDGEMENTS}

We would like to thank Liz Dooley and Clare Dooley for their support and assistance. We also wish to thank the following people for commenting on the draft protocol: Theresa Wrangham, Sushil Kabra, Segun Bello, Viviana Rodriguez and Taixiang Wu.

\section{REFEREN CES}

\section{Additional references}

\section{ATAGI 2008}

Australian Technical Advisory Group on Immunisation. The Australian Immunisation Handbook. Canberra:

Australian Government, 2008.

\section{Barrabeig 2011}

Barrabeig I, Rovira A, Rius C, Munoz P, Soldevila N, Batalla $\mathrm{J}$, et al.Effectiveness of measles vaccination for control of exposed children. Pediatric Infectious Disease Journal 2011; 30:78-80.

\section{Best 2011}

Best, Voss, Roberts, Freeman. Starship Children's Health Clinical Guideline: Measles - Infection Control Definitions \& Guidelines. www.adhb.govt.nz/ starshipclinicalguidelines/_Documents/Measles.pdf (accessed 30 April 2012).

\section{Birdsall 2009}

Birdsall HH. Antibodies. In: Mandell GL, Bennett JE, Dolin R editor(s). Mandell, Douglas, and Bennett's Principles and Practice of Infectious Diseases. 7th Edition. Philadelphia: Churchill Livingstone, 2009.

\section{Castillo-Solorzano 2011}

Castillo-Solorzano C, Reef S, Morice A, Andrus J, Ruiz $\mathrm{M}$, Tambini $\mathrm{G}$, et al.Guidelines for the documentation and verification of measles, rubella, and congenital rubella syndrome elimination in the region of the Americas. Journal of Infectious Diseases 2011;204(Suppl 2):683-9.

CDC 1998

Centers for Disease Control and Prevention. Measles, mumps, and rubella - vaccine use and strategies for elimination of measles, rubella, and congenital rubella syndrome and control of mumps: Recommendations of the Advisory Committee on Immunization Practices (ACIP). Morbidity and Mortality Weekly Report 1998;47(RR-8): $1-57$.

CDC 2011a

Centers for Disease Control and Prevention. Increased transmission and outbreaks of measles - European Region, 2011. Morbidity and Mortality Weekly Report 2011;60: 1605-10.

\section{CDC 2011b}

Centers for Disease Control and Prevention. General recommendations on immunization: Recommendations of the Advisory Committee on Immunization Practices (ACIP). Morbidity and Mortality Weekly Report 2011;60 (RR-2):1-61.

\section{CDNA 2009}

Communicable Diseases Network Australia. Measles: national guidelines for public health units. Canberra: Commonwealth Department of Health and Ageing, 2009.

\section{Delaporte 2011}

Delaporte E, Richard J, Wyler Lazarevic C, Lacour O, Girard M, Ginet C, et al.Ongoing measles outbreak, Geneva, Switzerland, January to March 2011. Euro Surveillance: European Communicable Disease Bulletin 2011; 16:10.

\section{DVD CDC 2011}

Division of Viral Diseases, National Centre for Immunization and Respiratory Diseases, CDC. Measles - United States, January - May 20, 2011. Morbidity and Mortality Weekly Report 2011;60(20):666-8.

\section{EMEA 1995}

European Medicines Agency. ICH Topic E 2 A - Clinical safety data management: definitions and standards for expedited reporting. www.emea.europa.eu/docs/en_ GB/document_library/Scientific_guideline/2009/09/ WC500002749.pdf (accessed 10 June 2010).

\section{Endo 2001}

Endo A, Izumi H, Miyashita M, Taniguchi K, Okubo O, Harada K. Current efficacy of postexposure prophylaxis against measles with immunoglobulin. Journal of Pediatrics 2001;138:926-8

Gonik 2011

Gonik B. Passive immunization: the forgotten arm of immunologically based strategies for disease containment. American Journal of Obstetrics and Gynecology 2011;205(5): 444.

\section{Gustavo 2008}

Gustavo H, Dayan M, Rota J, Bellini W, Redd S. Chapter 7: Measles (updated April 2009). Manual for the Surveillance 
of Vaccine-Preventable Diseases. Atlanta: Centers for Disease Control and Prevention, 2008.

\section{Heymann 2008}

Heymann DL. Control of Communicable Diseases Manual. Washington DC: American Public Health Association, 2008.

Higgins 2011

Higgins J, Green S (editors). Cochrane Handbook for Systematic Reviews of Interventions Version 5.1.0 [updated March 2011]. The Cochrane Collaboration, 2011. Available from www.cochrane-handbook.org 2011.

\section{Hoskins 2011}

Hoskins R, Vohra R, Vlack S, Young MK, Humphrey K, Selvey C, et al.Multiple cases of measles after exposure during air travel - Australia and New Zealand, January 2011. Morbidity and Mortality Weekly Report 2011;60:851.

\section{HPA 2011}

Health Protection Agency. Vaccination schedule: routine childhood immunisation schedule. www.hpa.org.uk/Topics/InfectiousDiseases/InfectionsAZ/ VaccineCoverageAndCOVER/VaccinationSchedule/ (accessed 2 January 2012).

\section{ID HPA 2009}

Immunisation Department. Measles. Immunoglobulin Handbook. UK: Health Protection Agency, 2009.

\section{Keller 2000}

Keller M, Stiehm E. Passive immunity in prevention and treatment of infectious diseases. Clinical Microbiology Reviews 2000;13(4):602-14.

King 1991

King G, Markowitz L, Patriarca P, Dales L. Clinical efficacy of measles vaccine during the 1990 measles epidemic. Pediatric Infectious Disease Journal 1991;10(12):883-8.

\section{Martin 2011}

Martin R, Wassilak S, Emiroglu N, Uzicanin A, Deshesvoi $S$, Jankovic $\mathrm{D}$, et al. What will it take to achieve measles elimination in the World Health Organization European Region: progress from 2003-2009 and essential accelerated actions. Journal of Infectious Diseases 2011;204(Suppl 1): $325-34$.

Moss 2009

Moss W, Scott S. The Immunological Basis for Immunization Series Module 7: measles. Geneva: World Health Organization, 2009.

\section{NZ MoH 2011}

New Zealand Ministry of Health. Immunisation Handbook 2011. Wellington: Ministry of Health, 2011.

\section{Ordman 1944}

Ordman C, Jennings C, Janeway C. Chemical, clinical, and immunological studies on the products of human plasma fractionation. XII. The use of concentrated normal human serum gamma globulin (human immune serum globulin) in the prevention and attenuation of measles. Journal of Clinical Investigation 1944;23(4):541-9.
Parker Fiebelkorn 2010

Parker Fiebelkorn A, Redd S, Gallagher K, Rota P, Rota J, Bellini W, et al.Measles in the United States during the postelimination era. Journal of Infectious Diseases 2010;202 (10): $1520-8$.

Ramsay 2009

Ramsay M, Manikkavasagan G, Brown K, Craig L. Post exposure prophylaxis for measles: revised guidance May 2009. UK: Health Protection Agency, 2009.

\section{Reading 2007}

Reading SA, Dimmock NJ. Neutralization of animal virus infectivity by antibody. Archives of Virology 2007;152: 1047-59.

\section{Sawyer 2000}

Sawyer L. Antibodies for the prevention and treatment of viral diseases. Antiviral Research 2000;47(2):57-77.

\section{Sheppeard 2009}

Sheppeard V, Forssman B, Ferson MJ, Moreira C, Campbell-Lloyd S, Dwyer DE, et al.The effectiveness of prophylaxis for measles contacts in NSW. New South Wales Public Health Bulletin 2009;20(5-6):81-5.

\section{Smithson 2010}

Smithson R, Irvine N, Hutton C, Doherty L, Watt A. Spotlight on measles 2010: ongoing measles outbreak in Northern Ireland following an imported case, SeptemberOctober 2010. Euro Surveillance: European Communicable Disease Bulletin 2010;15:43.

\section{Sniadack 2011}

Sniadack D, Mendoza-Aldana J, Jee Y, Bayutas B, LorenzoMariano K. Progress and challenges for measles elimination by 2012 in the Western Pacific Region. Journal of Infectious Diseases 2011;204(Suppl 1):439-46.

\section{Stokes 1944}

Stokes J, Maris E, Gellis S. Chemical, clinical, and immunological studies on the products of human plasma fractionation. XI. The use of concentrated normal human serum gamma globulin (human immune serum globulin) in the prophylaxis and treatment of measles. Journal of Clinical Investigation 1944;23(4):531-40.

Takimoto 2011

Takimoto N, Takahashi Y, Ishiyama A, Kishimoto K, Iwama R, Nakano M. Control of a measles outbreak by prohibiting non-vaccinated susceptible students from attending school in Akita Prefecture, Japan. Japanese Journal of Infectious Diseases 2011;64(4):309-11.

\section{UK DoH 2010}

UK Department of Health. Chapter 21: Measles - updated December 2010. Immunisation Against Infectious Disease "The Green Book". UK: Department of Health, UK, 2006.

Vainio 2011

Vainio K, Ronning K, Steen T, Arnesen T, Anestad G, Dudman S. Ongoing outbreak of measles in Oslo, Norway, January - February 2011. Euro Surveillance: European Communicable Disease Bulletin 2011;16:8. 
WHO 1999

World Health Organisation, Communicable Disease Surveillance and Response. WHO Guidelines for Epidemic Preparedness and Response to Measles Outbreaks. www.who.int/csr/resources/publications/ measles/whocdscsrisr991.pdf (accessed 10 June 2012).

WHO 2005

World Health Organization, UNICEF. GIVS: Global Immunization Vision and Strategy 2006-2015. Switzerland: WHO and UNICEF, 2005.

\section{WHO 2008}

World Health Organization. Progress towards reducing measles mortality and eliminating measles, WHO Eastern Mediterranean Region, 1997-2007. Weekly Epidemiological Record 2008;83(11):97-104.

WHO 2009a

World Health Organization. Measles vaccines: WHO position paper. Weekly Epidemiological Record 2009;84(35): $349-60$.

\section{WHO 2009b}

World Health Organization. Global reductions in measles mortality 2000-2008 and the risk of measles resurgence.
Weekly Epidemiological Record 2009;84(49):509-16.

WHO 2011

World Health Organisation. Global health observatory data repository: World Health Statistics - selected infectious diseases, number of reported cases. www.apps.who.int/ ghodata/ (accessed 10 June 2012).

\section{WHO 2012a}

World Health Organization. Progress in global measles control, 2000-2010. Weekly Epidemiological Record 2012;87 (5):45-52.

\section{WHO 2012b}

World Health Organization. WHO Vaccine Preventable Diseases Monitoring System 2011 Global Summary. www.apps.who.int/immunization_monitoring/en/ globalsummary/countryprofileselect.cfm (accessed 21 February 2012).

Zingher 2005

Zingher A, Mortimer P. Convalescent whole blood, plasma and serum in the prophylaxis of measles: JAMA, 12 April, 1926; 1180-7. Reviews in Medical Virology 2005;15(6): 407-18.

* Indicates the major publication for the study

\section{A P P E N D I C E S}

\section{Appendix I. CENTRAL and MEDLINE search strategy}

1 exp Measles/

2 exp Measles virus/

3 measles.tw.

4 (rubeola or rubeolla).tw.

5 or/ $1-4$

6 exp Immunoglobulins/

7 (immunoglobulin* or immuno-globulin* or immun* globulin*).tw,nm.

8 (gammaglobulin* or gamma-globulin* or gamma globulin*).tw,nm.

9 exp Immunization, Passive/

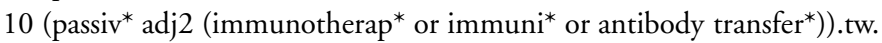

11 Post-Exposure Prophylaxis/

12 ((post exposur* or post-exposur* or postexposur*) adj2 (prophyla* or prevent*)).tw.

13 or/6-12

145 and 13 


\section{Appendix 2. CENTRAL and MEDLINE filter for study type}

We will combine the following filter for non-randomised prospective intervention studies (not before and after and not time-series studies) with the Cochrane Highly Sensitive Search Strategy for identifying randomized trials (Higgins 2011).

1. exp Cohort Studies/

2. Epidemiologic Studies/

3. Intervention Studies/

4. Evaluation Studies/

5. Program Evaluation/

6. Random Allocation/

7. Clinical Trial/

8. Single-Blind Method/

9. Double-Blind Method/

10. Control Groups/

11. Pilot Projects/

12. controlled clinical trial.pt.

13. clinical trial.pt.

14. comparative study.pt.

15. multicenter study.pt.

16. evaluation studies.pt.

17. Comparative Study/

18. Multicenter Study/

19. Follow-Up Studies/

20. Prospective Studies/

21. (cohort adj (study or studies)).tw.

22. cohort analy*.tw.

23. cohort*.tw.

24. ("follow up" or follow-up) adj (study or studies or assessment)).tw.

25. (observational adj (study or studies)).tw.

26. longitudinal.tw.

27. prospective.tw.

28. ((single or double* or triple* or treb*) and (blind* or mask*)).tw.

29. trial*.tw.

30. placebo.tw.

31. groups.tw.

32. ("pre test" or pretest or pre-intervention or preintervention or "pre intervention" or "post test" or posttest or post-intervention or postintervention or "post intervention").tw.

33. (pre adj5 post).tw.

34. ((evaluat* or intervention or interventional or treatment) and (control or controlled or study or studies or program* or comparison or comparative or "usual care")).tw.

35. ((intervention or interventional or process or program) adj8 (evaluat* or effect* or outcome*)).tw.

36. (program or programme or secondary analyse*).tw.

37. (quasi-experiment* or Quasiexperiment* or "quasi random*" or quasirandom* or "quasi control*" or quasi control* or ((quasi* or experimental) adj3 (method* or study or studies or trial or design*))).tw.

38. random*.tw.

39. (study adj3 aim*).ab.

40. "our study".ab.

41. multivariate.ab.

42. compared.ab.

43. intervention*.ti.

44. pilot.ti.

45. (multicentre or multicenter or multi-centre or multi-center).ti.

46. controlled.ti.

Post-exposure passive immunisation for preventing measles (Protocol)

Copyright ( 2012 The Cochrane Collaboration. Published by John Wiley \& Sons, Ltd. 
47. (rat or rats or cow or cows or chicken* or horse or horses or mice or mouse or bovine or animal*).ti.

48. exp animals/ not humans.sh.

49. (or/1-46) not (47 or 48)

\section{H I S T O R Y}

Protocol first published: Issue 9, 2012

\section{CONTRIBUTIONSOFAUTHORS}

Dr Megan Young (MY) and Prof Graeme Nimmo (GN) will obtain copies of the studies and select studies for inclusion in the review. MY and Prof Allan Cripps (AC) will extract the data and assess the risk of bias in the studies.

MY and Dr Mark Jones (MJ) will enter the data.

MJ will analyse the data and together with MY will interpret the analysis.

All authors will complete the final review.

\section{DECLARATIONSOF INTEREST}

Dr Megan Young is a public health physician in Queensland, Australia who is involved in the public health management of measles.

\section{SOURCES OF SUPPORT}

\section{Internal sources}

- Griffith University, Australia.

In-kind employee time

- University of Queensland, Australia.

In-kind employee time

- Queensland Health, Australia.

In-kind employee time

\section{External sources}

- No sources of support supplied 